\title{
Intravesical oxybutynin for spinal cord injury patients
}

\author{
Suzanne M Szollar and SM Lee \\ Spinal Cord Injury Center, University of California, San Diego, CA 92161, USA
}

\begin{abstract}
The treatment of choice for the failure to restore neurogenic bladder dysfunction, managed with clean intermittent catheterization program with incontinence, is anticholinergic medication. The goal is to increase bladder capacity, and decrease intravesical pressure in order to maintain continence between catheterizations. The most commonly used anticholinergic medication in the United States is Oxybutynin. Previous clinical studies have shown that $61 \%$ of the patients on oral Oxybutynin report adverse systemic side effects and are noncompliant in taking it. In $48 \%$ of the patients, possibly due to their noncompliance, oral Oxybutynin Hydrochloride is ineffective. ${ }^{1,2}$

In this study, 13 spinal cord injured patients with a failure to restore bladder dysfunction were treated with intravesical Oxybutynin. Nine patients improved on the regimen while one patient underwent surgical bladder augmentation. Postoperatively, this patient continued to be incontinent between catheterizations. Reinstitution of intravesical Oxybutynin stabilized the patient's bladder with clean intermittent catheterization without further intervention.

After 3 months post instillation in the urodynamic studies, the mean bladder capacity increased, the mean volume at first contraction increased and the leak point pressure decreased. The decrease in leak point pressure was statistically significant. Leak point pressure is one of the most important parameters we monitor to preserve upper urinary tract function. We recommend the trial of intravesical Oxybutynin for patients who fit the criteria of the protocol prior to surgical bladder augmentation.
\end{abstract}

Keywords: neuropathic bladder; clean intermittent catheterization (CIC); Oxybutynin Hydrochloride; intravesical instillation; medical bladder augmentation

\section{Introduction}

Voiding dysfunction can pose a difficult management problem in clinical care. The storage and elimination of urine depends on a complex central control system that coordinates autonomic and somatic efferent activity to various effector organs (the bladder, the urethra and the striated muscles of the pelvic floor). There are three major muscle structures responsible for the storage and the elimination of urine from the bladder.

The detrusor muscle receives primarily parasympathetic cholinergic innervation; the external sphincter muscle has sympathetic, parasympathetic and somatic fibers; and the bladder outlet or 'internal sphincter' receives mainly alpha-adrenergic sympathetic innervation. Activation of the parasympathetic pathways of the detrusor via the parasympathetic pathways and inhibition of the internal urethral sphincter via the somatic input are essential neural events initiating the release of the urine. Activation of the detrusor muscle occurs via a spino-bulbo-spinal pathway. Relaxation of the urinary sphincter is produced by inhibitory mechanisms in the sacral spinal cord. ${ }^{3,4}$

Urodynamic studies measure bladder capacity,

Correspondence: SM Szollar bladder volume at the first urge to void and the leak point pressure. There are two major bladder dysfunctions based on urodynamic studies: failure to empty and failure to store. ${ }^{5}$

The management of choice for the failure to empty bladder dysfunction is clean intermittent catheterization (CIC). Patients with failure to store bladder dysfunction experience urgency (in case of incomplete spinal cord injury), frequency and incontinence due to the small bladder capacity. It can occur due to increased detrusor activity or decreased outlet resistance. On urodynamic studies, this bladder has a capacity of less than $300 \mathrm{cc}$. The first urge to void appears at a volume of less than $150 \mathrm{cc}$, when the leak point pressure is $45 \mathrm{cmH}_{2} \mathrm{O}$. Patients will often restrict their fluid intake and decrease their social activity. The prolonged high pressure on the upper urinary tracts may result in renal function deterioration. The treatment is aimed to decrease intravesical pressure and increase bladder capacity, thus, creating a large capacity bladder which can be managed with CIC. The medical treatment of choice is anticholinergic medication. Oxybutynin Hydrochloride is one of the most commonly used medications in the United States. The maximum recommended dose is $5 \mathrm{mg}$ three times/day. 
Its usefulness is limited by its side effects: xerostomia and constipation. ${ }^{6}$ In case of failure of the medical management, surgical intervention with bladder augmentation is the alternative treatment to restore upper urinary tracts.

\section{Methods}

Thirteen patients with failure to store neurogenic bladder dysfunction on CIC were included in the study. Selection criteria were poor tolerance to oral Oxybutynin and/or unsatisfactory response. Poor tolerance was defined as the inability to tolerate $5 \mathrm{mg}$ Oxybutynin three times/day, which is the maximum recommended oral dose. Unsatisfactory response was defined as incontinence on the maximum recommended oral dose. The selection criteria for the clean intermittent catheterization program was adequate dexterity and cognitive function. The mean age of the patients was 41 years with a range of 20-68 years. Levels of spinal cord injury ranged from $\mathrm{C} 5$ to $\mathrm{L} 1$. The main interval after injury was 8.7 years and ranged from one year to 26 years. Baseline laboratory studies included: serum creatinine, BUN, 24 h urine creatinine clearance, upper tract imaging and videourodynamic studies. Videourodynamic studies were performed utilizing a Life Tech Model 1406 machine. A 9F dual lumen urethral catheter was utilized. The needle electrode was positioned in the external sphincter muscle under transrectal ultrasound guidance. The abdominal pressure was monitored with a rectal balloon. The bladders were filled with $14 \%$ Conray contrast at $50 \mathrm{ml} / \mathrm{min}$. Detrusor pressure, intraabdominal pressure, subtracted detrusor pressure, and sphincter activity was recorded. If all of the baseline laboratory tests, except videourodynamic studies, were within normal limits the patients were offered inclusion in the study. The patients who agreed to participate were instructed to dilute $5 \mathrm{mg}$ crushed Ditropan in $30 \mathrm{ml}$ saline solution and to instill the solution into the bladder following catheterization three times/day. The Oxybutynin was left in the bladder until the next catheterization. Clinical progress was monitored with scheduled outpatient clinic visits. At the end of 3 months on the regimen, videourodynamic studies were repeated.

For statistical analysis, paired samples of $t$ test were used.

\section{Results}

$69 \%$ of the patients included in the study reported continence. $50 \%$ of the patients who failed to respond to oral Oxybutynin Hydrochloride reported continence. $80 \%$ of the patients who were converted to instillation because of poor tolerance reported continence. The instillation's were tolerated well. One patient required surgical bladder augmentation due to failure to respond to intravesical Oxybutynin Hydrochloride. Following augmentation cystoplasty he continued to be incontinent between catheterizations. Incontinence ceased following reinstitution of intravesical Oxybutynin. Repeated videourodynamic parameters revealed improvement in 3 months. The mean bladder capacity (Figure 1) improved from a mean of $344 \mathrm{cc}$ with a standard error of 36.2 to $400 \mathrm{cc}$, with a standard error of 56.32. The mean leak point pressure (Figure 2) decreased from $65 \mathrm{~cm}$ of $\mathrm{H}_{2} \mathrm{O}$ with a standard error of 6.55 to $47 \mathrm{~cm}$ of $\mathrm{H}_{2} \mathrm{O}$ with a standard error of 7.17 . The mean volume at first contraction (Figure 3) increased from $167 \mathrm{cc}$ with a standard error of 24.17 to $184 \mathrm{cc}$ with a standard error of 25.45 .

The improvement in bladder capacity and the volume at first urge to void was not statistically



Figure 1 Mean bladder capacity

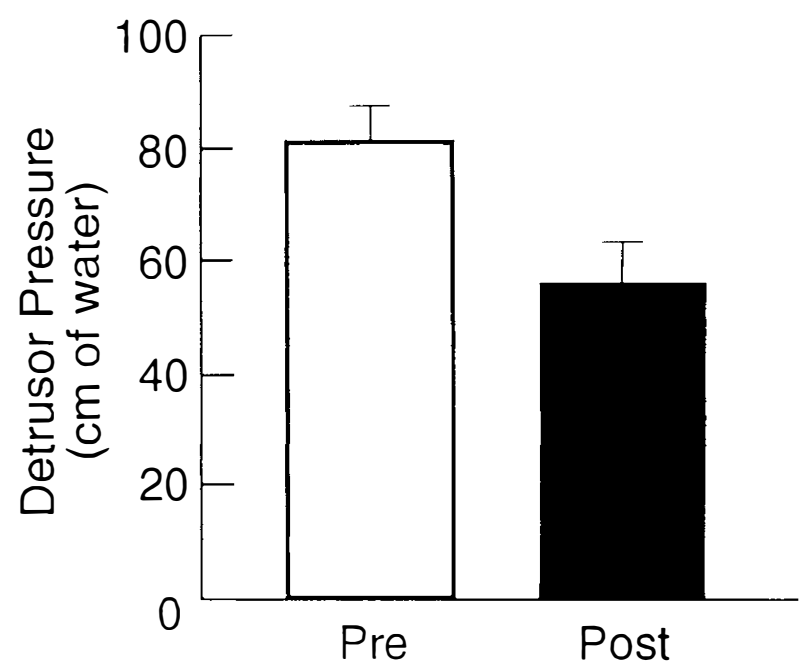

Figure 2 Mean leak point pressure 


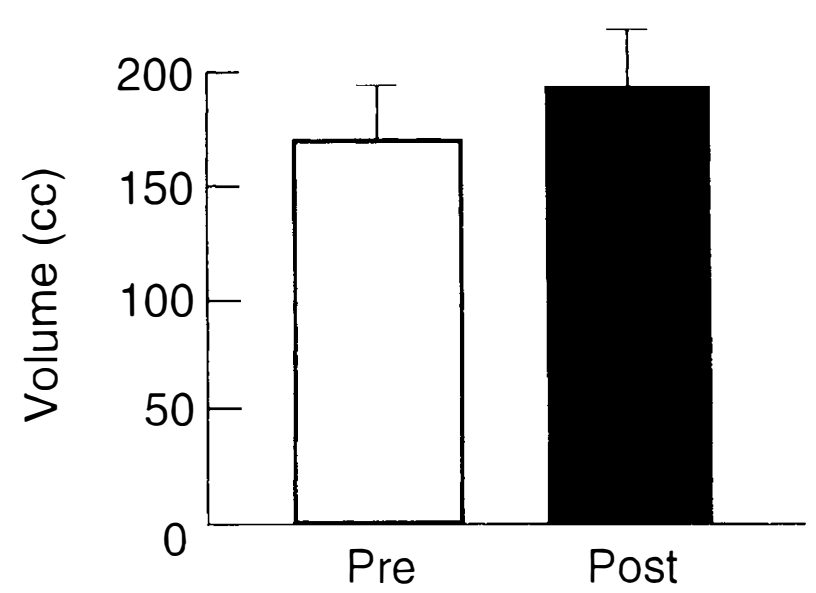

Figure 3 Mean volume at first contraction

significant. The decrease in leak point pressure, which is the most crucial parameter to monitor in protecting upper urinary tract function, was found to be statistically significant.

\section{Discussion}

Oxybutynin Hydrochloride is a synthetic tertiary amine which is chemically and pharmacologically similar to some antispasmodic local anesthetics and antihistamine compounds. It exerts a direct spasmolytic action and an antimuscarinic (atropine-like) action on smooth muscle. The spasmolytic effect of the drug has been demonstrated on the detrusor muscle of the bladder, the small intestine and the colon. In animal studies, the drug has shown moderate antihistaminic, some local anesthetic, mild analgesic, midriatic, and antisiologogue activity. Studies using radiolabeled oxybutynin indicate that the drug undergoes enterohepatic circulation and is excreted in urine and feces. It is widely used to treat detrusor instability. Its effectiveness is limited by intolerance to oral administration, due to systemic side effects. In an effort to mitigate the side effects associated with oral anticholinergic agents and provide another option to patients who would otherwise be offered an augmentation cystoplasty, attention has focused on the use of intravesical agents to treat failure to store bladder dysfunction. The studies reported in the literature ${ }^{7-13}$ examined the short-term response to intravesical oxybutynin, and only one examined long term effects. ${ }^{14}$ All of the investigators claimed to have less systemic side effects with intravesical oxybutynin than with oral administration. It has been theorized that intravesical oxybutynin is not absorbed into the bloodstream, and its efficacy results from a profound local effect alone. ${ }^{13}$ However, Massad et al. ${ }^{15}$ reported significant plasma concentrations within $5 \mathrm{~min}$ following intravesical instillations, higher than with the oral administration. Peak plasma concentrations are achieved in about $1 \mathrm{~h}$ after which time the levels rapidly decrease. The hepatic metabolite seems to be responsible for the side effects which appear at a lower plasma concentration with oral administration. Intravesical instillations circumvent the portal circulation.

Bonney et $a l^{16}$ investigated topical effects of intravesical oxybutynin and found no evidence of drug-related mucosal or bladder wall change. The studies in the literature, a total of six, report beneficial effects from intravesical oxybutynin. There are no reports that we are aware of regarding statistically significant improvement in leak point pressure. Most of the investigators instilled the solutions only for shorter periods of time $(30-180 \mathrm{~min})$. There were no local side effects from leaving the solution for as long as the next catheterization and technically it is much more simple. It also improves patient compliance, therefore, we decided not to institute extra catheterizations in our protocol. Mizunga et al ${ }^{14}$ filtered and buffered the solution prior to instillations. In our study the patients were instructed how to prepare the solution with the crushed oxybutynin and it was effective without side effects.

\section{Conclusion}

Our data supports other researchers findings about the effectiveness of intravesical Oxybutynin Hydrochloride. Patients who are on a CIC program can easily incorporate intravesical instillations into their catheterization routine. Oxybutynin Hydrochloride is chemically and pharmacologically similar to some antispamodic and local anesthetic compounds. It exerts a direct spasmolytic action on smooth muscle. The spasmolytic effect of the drug has been demonstrated on the detrusor muscle of the bladder, the small intestine, and the colon. It was shown to cause no longterm complications locally in the bladder mucosa or systematically. It increases bladder capacity, decreases intravesical pressure, and reduces leak point pressure, (detrusor pressure at which urinary leakage occurs), ${ }^{24}$ restoring the upper urinary tracts without surgical intervention. It is recommended for patients on CIC, and can be successfully utilized to augment intestinal bladders, ${ }^{10}$ or augmented bladders. The technique is simple, and although the solution is not yet available in the United States patients can make their own solution. This treatment certainly should be offered to patients who have failed other alternative conservative bladder management prior to surgical intervention.

\section{Acknowledgements}

This study was supported by the Department of Veterans Affairs Medical Center, San Diego, CA 92161. 


\section{References}

1 Thompson IM, Lauvetz R. Oxybutynin in bladder spasm, neurogenic bladder and enuresis. Urology 1976; 8: 452 - 454.

2 Holmes DM, Montz FJ, Stanton SL. Oxybutynin versus propantheline in the management of detrusor instability. A patient-regulated variable dose trial. $B r \quad J O b$ Gyn 1989; 96: $607-612$.

3 Thuroff JW, Bunke B et al. Randomized, double-blind, multicenter trial on treatment of frequency, urgency and incontinence related to detrusor hyperactivity: Oxybutynin versus propantheline versus placebo. J Urol 1991; 145: 813-817.

4 Brooks ME, Braf ZF. Oxybutynin Chloride (Ditropan)-clinical uses and limitations. Paraplegia 1980; 18: 64-68.

5 Gajewki JB, Awad SA. Oxybutynin versus propantheline in patients with multiple sclerosis and detrusor hyperreflexia. J Urol 1986; 135: $966-968$.

6 Moisey CU, Stephenson TP, Brendler CB. The urodynamic and subjective results of treatment of detrusor instability with oxybutynin chloride. Br J Urol 1980; 52: 472-475.

7 Gillenwater JY. Adult and pediatric urology. 2nd ed. St Louis: Mosby Year Book. 1991.

8 Greenfield SP, Fera M. The use of intravesical oxybutynin chloride in children with neurogenic bladder. J Urol 1991; 146: $532-534$.

9 Massad CA, Kogan BA, Trigo-Rocha FE. The pharmacokinetics of intravesical and oral oxybutynin chloride. J Urol 1992; 148: 595-597.

10 Mizunga $\mathrm{M}$ et al. Intravesical instillation of oxybutynin hydrochloride therapy for patients with a neurogenic bladder. Poster presentation, Kobe, Japan 1994, Annual conference of the International Paraplegia Society.

11 Brendler CB, Radebaugh LC, Mohler JL. Topical oxybutynin chloride for relaxation of dysfunctional bladders. J Urol 141: $1350-1351$.

12 Kasabian NG, Vlachiotis JD et al. The use of intravesical oxybutynin chloride in patients with detrusor hypertonicity and detrusor hyperreflexia. J Urol 1994; 151: 944-945.
13 Weese DL, Roskamp DA, Leach GE, Zimmern PE. Intravesical oxybutynin chloride: experience with 42 patients. Urol 1993; 41: $527-530$.

14 Mohler JL. Relaxation of intestinal bladders by intravesical oxybutynin chloride. Neuro Urol 1990; 9: 179-187.

15 Mattiasson A, Ikstrom B, Andersen KB. Effects of intravesical instillation of verapamil in patients with detrusor hyperactivity. Neuro Urol Urodyn 1987; 6: 253-254.

16 Brendler CB, Radebaugh LC, Molher JL. Topical oxybutynin chloride for relaxation of dysfunctional bladders. J Urol 1989; 141: $1350-1352$

17 Madersbacher H, Jilg G. Control of detrusor hyperreflexia by the intravesical instillation of oxybutynine hydrochloride. Paraplegia 1991; 29: $84-90$

18 Dykstra DD, Sidi AA et al. Effects of botulinum a toxin on detrusor-sphincter dyssynergia in spinal cord injury patients. $J$ Urol 1988; 139: 919-922.

19 Fall M, Ohlsson BL, Carlsson CA. The neurogenic overactive bladder, classification based on urodynamics. Br J of Urol 1989; 64: $368-373$.

20 Perkash I. Long-term urologic management of the patient with spinal cord injury. Urol Clinics of N Amer, 1993; 20-3: 423-434.

21 deGroat W, Booth AM. Physiology of the urinary bladder and urethra. Annals of I M 1980; 92(Part 2): 312-315.

22 deGroat W, Kawatani M. Neurol Control of the urinary bladder: possible relationship between peptidergic inhibitory mechanisms and detrusor instability. Neuro and Urol 1985; 4: 285-300.

23 Bonney W, Robinson R, Theobald RJ. Topical effect of intravesical oxybutynin. J of Urol 1993; 150: $1522-1525$.

24 Juma S, Mostavfavi M, Joseph A. Sphincterotomy: long term complications and warning signs. Neurology and Urodynamics 1995; 14: $33-41$. 